\title{
Terrestrial Magnetism and \\ Atmospheric Electricity
}

VOIUME XXIV

JUNE, I9I9

NUMBER 2

\section{THE VERTICAL-INTENSITY VARIOMETER. \\ By George HarTNELI.}

For the purpose of recording the variations of the three magnetic elements, declination $(D)$, horizontal intensity $(H)$, and vertical intensity $(Z)$, magnetic observatories use three instruments collectively called a magnetograph. One instrument called the $D$-variometer or sometimes the unifilar variometer records variations in declination; its magnet is suspended horizontally with north end of the magnet north in the magnetic meridian, by a single fibre of silk or quartz. Another, with north end of the magnet either east or west, is suspended in a horizontal plane by either a single fibre of quartz, or by a bifilar suspension of silk fibres; it records variation of the horizontal intensity and is called the $H$-variometer. In the case of the bifilar suspension it is sometimes called the bifilar variometer. The third instrument of the magnetograph, the $Z$ - or vertical-intensity variometer, records variations in the vertical intensity. It consists essentially of a magnet supported on a horizontal axis, with freedom to move in a vertical plane. In construction the support may be an agate knife-edge resting on a horizontal plane of polished agate, or a pair of steel points resting on an agate plane or in shallow agate cups. In still another type, the magnet is attached to a quartz plate to which horizontal suspension fibres of quartz are fused. We shall discuss some of the characteristic features of the $Z$-variometer in which the magnet is supported by an agate knife-edge or by a pair of steel points resting on a horizontal plane, either form of support being called a knife-edge for brevity.

Referring to Fig. 1 let $x$ and $z$ be the coordinates of the center of gravity of the magnet system, $x$ being positive toward the magnetic north, and $z$ being positive vertically upward. The coordinate $y$, positive toward the east, is 0 . The origin of co- 
ordinates is at $O$, where the knife-edge touches the plane. Let $h$ be the distance $O O^{\prime}$ perpendicular to the axis of the magnet; $c$,

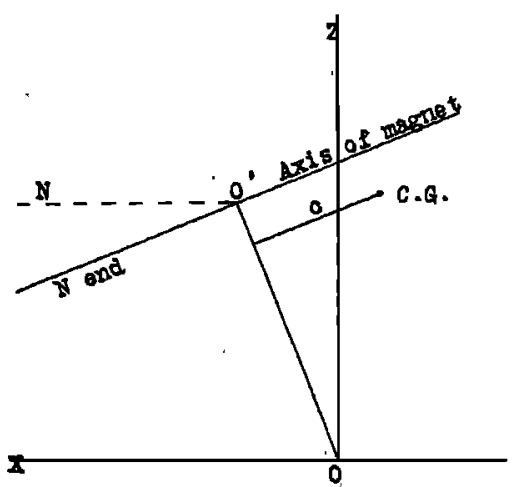

FIG. 1. parallel to the axis of the magnet, the distance from $O^{\prime}$ to the center of gravity. $\theta$ is the angle which the magnet makes with the horizontal and is positive when the north end dips downward. Also let $m$ be the mass of the magnet; $M$, the magnetic moment; $H$ and $Z$, horizontal and vertical intensities; $g$, the acceleration of gravity; and $k$ the radius of gyration around the center of gravity. Referred to this set of rectangular axes, $H$ is positive while $Z$ and $g$ are negative; the force in the $y$ direction is 0 , when the magnet moves in a vertical plane in the magnetic meridian. In the actual construction of the variometer, the magnet is provided with two poises or small weights. One moves along $h$ and alters the sensitivity by raising or lowering the center of gravity, and is here called the "sensitivity poise"; the other moves along $c$ and is called the "balancing poise."

To determine the equation of motion we shall use Lagrange's equation:

$$
\frac{d}{d t} \frac{d L}{d \dot{\xi}}-\frac{d L}{d \xi}=0
$$

where $L$, the kinetic potential, is $T-V, T$ being the kinetic energy and $V$ the potential energy, and $\xi$ is any coordinate desired.

In our problem the kinetic energy is

$$
T=\frac{m}{2}\left(\dot{x}^{2}+\dot{z}^{2}+k^{2} \dot{\theta}^{2}\right) \text { and } V=m g z-M(H \cos \theta+Z \sin \theta)
$$

The coordinates of the center of gravity are

$$
x=h \sin \theta-c \cos \theta, \text { and } z=h \cos \theta+c \sin \theta
$$

Substituting in $T$ and $V$ and reducing

$$
\begin{aligned}
T= & \frac{m}{2}\left(h^{2}+c^{2}+k^{2}\right) \dot{\theta}^{2} \text { and } V=m g(h \cos \theta+c \sin \theta)- \\
& M(H \cos \theta+Z \sin \theta)
\end{aligned}
$$


Whence

$$
\begin{aligned}
L= & T-V=\frac{m}{2}\left(h^{2}+c^{2}+k^{2}\right) \dot{\theta^{2}}-m g(h \cos \theta+c \sin \theta)- \\
& M(H \cos \theta+Z \sin \theta)
\end{aligned}
$$

Using this value of $L$ in (1), $\theta$ being the coordinate desired, we get the equation of motion:

$$
K_{v} \ddot{\theta}+(M H-m g h) \sin \theta+(m g c-M Z) \cos \theta=0
$$

where $K_{0}=m\left(h^{2}+c^{2}+k^{2}\right)$.

Equation (2) describes the action of two groups of impressed forces-namely, the magnetic forces and the gravity forces-on the motion of the magnet system. Among other ways, it could have been derived from considering how the two groups of forces are, so to speak, paired off against each other. Under ordinary operating conditions the magnetic turning-moment $M H \sin \theta$ is considerably larger than the gravity turning-moment $m g h \sin \theta$, and the resultant is a restoring force tending to keep the magnet level. The tendency of the magnetic turning-moment $M Z \cos \theta$ to upset the magnet is almost completely counterbalanced by the gravity turning-moment $m g c \cos \theta$. The resultant of all the turning-moments, when not zero, imparts motion to the magnet system and $=K_{v} \ddot{\theta}$.

Moreover, equation (2) represents an oscillation around a position of equilibrium. To determine this position, place $\ddot{\theta}$ in $(2)=0$, and the condition to be fulfilled for equilibrium is

$$
\tan \theta_{0}=\frac{M Z-m g c}{M H-m g h}
$$

Or in case the instrument is not in the magnetic meridian as we have assumed

$$
\tan \theta=\frac{M Z-m g c}{M H \cos A-m g h}
$$

where $A$ is the azimuth of the vertical plane containing the magnet. The magnet will be level or balanced, that is $\theta$ will be 0 , when $M Z-m g c=0$ and this will be true for all azimuths or for all values of $A$. However, if ( $M Z-m g c$ ) is not exactly 0 , as would probably be the case, $\theta$ would change as $A$ changes.

If $c=h=0$, that is, if the magnet were supported at its center of gravity, as would be true in the case of a perfectly symmetrical dip needle provided with conical pivots resting in agate cups such as is used at sea for determining the dip, $I$, 


$$
\tan \theta=\frac{Z}{H \cos A}
$$

When $A=0, \tan \theta=\frac{Z}{H}=\tan I$; when $A=90^{\circ}, \tan \theta=\infty$, and $\theta=90^{\circ}$, the north end of the magnet pointing vertically downward.

If the variometer is oriented in the magnetic prime-vertical,

$$
A=90^{\circ}, \text { and } \tan \theta=\frac{M Z-m g c}{-m g h}
$$

In this position, the magnet will be unstable unless the "sensitivity poise" is lowered until the center of gravity is below the point of support, that is, below the surface of the agate plane. If $M=0$, that is if the magnet were merely a non-magnetic body, it would become simply a compound pendulum.

In order to determine the periods of the magnet for different azimuths, let the magnet be displaced from the position of equilibrium $\theta_{0}$ by a small angle $d \theta$, so that $\theta$ becomes $\theta_{0}+d \theta$. Substituting in (2) and introducing $A$ and rearranging we get

$K_{v} \ddot{\theta}+\left[(M H \cos A-m g h) \cos \theta_{0}-(m g c-M Z) \sin \theta_{0}\right] \sin d \theta$

$$
+\left[(M H \cos A-m g h) \sin \theta_{0}+(m g c-M Z) \cos \theta_{0}\right] \cos d \theta=0
$$

The period, that is, the time required to describe half a complete vibration will be

$$
T^{2}{ }_{v}=\frac{\grave{\pi}^{2} K_{v}}{(M H \cos A-m g h) \cos \theta_{0}-(m g c-M Z) \sin \theta_{0}}
$$

When the magnet is level, $m g \epsilon-M Z=0, \theta_{0}=0$ and the period is

$$
T^{2}{ }_{v}=\frac{\pi^{2} K_{v}}{M H \cos A-m g h}
$$

If the magnet also lies in the magnetic meridian, $A=0$, and the period is

And if $h$ is 0 ,

$$
T^{2}{ }_{v}=\frac{\pi^{2} K_{v}}{M H-m g h}
$$

$$
T_{v}^{2}=\frac{\pi^{2} K_{v}}{M H}
$$

If $A=90^{\circ}$ and $\theta_{0}=0, T^{2}{ }_{v}=\frac{\pi^{2} K_{v}}{-m g h}$, the magnet would be unstable unless the "sensitivity poise" were lowered until the center of gravity were below the point of support and $h$ made 
negative. It will be seen that $H$ does not appear in this equation; in other words the horizontal intensity, being perpendicular to the plane in which the magnet moves, has no effect on its motion and thus no effect on its period and sensitivity.

When $h=c=0$,

If $A=0$,

$$
T^{2}{ }_{v}=\frac{\pi^{2} K_{v}}{M H \cos A \cos \theta_{0}+\overline{M Z \sin \theta_{0}}}
$$

$$
\begin{aligned}
& T^{2}{ }_{v}=\frac{\pi^{2} K_{v}}{M H \cos \theta_{0}+M Z \sin \theta_{0}}=\frac{\pi^{2} K_{v}}{M H \cos I+M Z \sin I} \\
& =\frac{\pi^{2} K_{v} \sin I}{M Z}=\frac{\pi^{2} K_{v} \cos I}{M H}=\frac{\pi^{2} K_{v}}{M F}
\end{aligned}
$$

where $F=\sqrt{H^{2}+Z^{2}}=$ the total magnetic intensity. For $A=90^{\circ}$, and so $\theta_{0}=90^{\circ}$,

Moreover,

$$
T_{z}^{2}=\frac{\pi^{2} K_{v}}{M Z}
$$

$$
\frac{M Z}{M F}=\sin I=\frac{\pi^{2} K}{T_{z}^{2}} \div \frac{\pi^{2} K}{T_{F}^{2}}=\frac{T^{2} F}{T_{z}^{2}}
$$

From equation (13) the dip $I$ could be obtained by determining the periods of the magnet when oscillating in and perpendicular to the magnetic meridian. Equations (10), (11), (12), and (13) are simply illustrations of the law that the square of the period of a vibrating body varies inversely as the force. In all the foregoing expressions for $T$ and $\theta$, the denominators must be positive, otherwise the periods will be imaginary, and the magnet will not be stable and will not oscillate.

Returning to equation (3) which specifies the position of . equilibrium of the magnet when in the magnetic meridian, that position will be when the magnet is level if

$$
M Z=m g c
$$

To get a concrete idea of the magnitude of $c$ at a station where $Z=0.554$ and $g=981$, let us take a magnet which we shall call magnet $J$, having mass 61.0 and the magnetic moment 750.0 , all quantities being expressed in C.G.S. units. With these values $c$ will be $0.0069 \mathrm{~cm} .=0.069 \mathrm{~mm} .=69 \mu$.

Differentiating equation (3) with reference to $\theta$ and $Z, \theta$ being a small angle, and solving

$$
d Z=\frac{M H-m g h}{M} d \theta
$$


Let $\epsilon$ be the scale value, and $n$ the number of divisions through which the magnet is deflected, and $\theta_{r}$ the value in radian of one millimeter on the magnetogram.

Then

and

$$
d Z=n \epsilon=\left(\frac{M H-m g h}{M}\right) n \theta_{r}
$$

$$
\varepsilon=\left(\frac{M H-m g h}{M}\right) \theta_{r}
$$

For a concrete idea of $h$, let us compute $h$ from (15) for the usual scale-value of $5 \gamma$ per $\mathrm{mm}$. on the magnetogram, $H$ being taken as 0.192 . For example, if the magnet $J$ were mounted as a $Z$-variometer at the distance $R=150 \mathrm{~cm}$. from the drum of the recording apparatus, $h$ would be $0.00053 \mathrm{~cm} .=0.0053 \mathrm{~mm} .=5 \mu$ approximately. If it were mounted at the distance $R=230 \mathrm{~cm}$. $h$ would be $-0.00047 \mathrm{~cm} .=-0.0047 \mathrm{~mm} .=-5 \mu$ approximately. It will thus be seen that both $h$ and $c$ are exceedingly small quantities. For $R=150 \mathrm{~cm} ., h$ is positive, and the center of gravity is slightly above the knife-edge; for $R=230 \mathrm{~cm}$., $h$ is negative, and the center of gravity is slightly below the knife-edge. If desired, we could compute from (15) the distance at which to place the variometer for a given scale-value when $h=0$; thus $R=\frac{H}{20 \epsilon}$ since $\theta_{r}=\frac{1}{20 R}$. For any azimuth the scale-value would be $\epsilon=\frac{M H \cos A-m g h}{M}$, and for a $Z$-variometer oriented in the magnetic prime-vertical $\epsilon=\frac{-m g h}{M} \theta_{r}$, the center of gravity being slightly below the knife-edge. Under these circumstances and for a distance of $R=230 \mathrm{~cm}$., $h=-0.0029$ $\mathrm{cm} .=-0.029 \mathrm{~mm} .=-29 \mu$.

If $h$ were 0 , (15) would become

$$
\epsilon=H \theta_{r}
$$

from which it follows that since $M H$ is considerably larger than $m g h$, the chief factors determining the scale-value of a variometer in the magnetic meridian are the horizontal intensity and the distance.

At a station where there is a secular decrease in $H$, the scale-value would be expected to decrease, that is the variometer would become more sensitive in the course of time. However, it will be shown later that a rolling magnet is less sensitive than a pivoting magnet; and from (15) it is clear that a decrease in $h$ will also decrease the sensitivity. So if the variometer loses sensi- 
tivity, the probable explanation is that the loss of sensitivity due to the wearing away and rounding off of the knife-edge more than offsets the increase of sensitivity due to the secular decrease in $H$. These remarks may be made sufficiently clear by considering the pivoting magnet only. For a small change $d \epsilon$ in scalevalue we have $d \varepsilon=\frac{M d H-m g d h}{\mathrm{M}}$. If the scale-value is constant, $d H$ being negative, $-d h=\frac{M d H}{m g}$. Since $\frac{M}{m g}$ is a small fraction, the decrease in the length $h$ is sufficient to offset the decrease in scale-value due to decrease in $H$, is only a small fraction of the decrease in $H$. Hence while the distance and the horizontal intensity largely determine the actual scale-value, changes in the length $h$ may, under some circumstances, be the controlling factor in causing scale-value changes.

In actual construction the knife-edge is equidistant from the geometrical center of the magnet, and the center of gravity of the magnet system is below the knife-edge or plane and the magnet is balanced and adjusted to the desired sensitivity by moving the poises. Let $m^{\prime}$ be the mass of the "sensitivity poise," $h$ ' its distance from the supporting plane measured perpendicular to the axis of the magnet; and let $l$ be the distance of the center of gravity below the plane. Also let $m^{\prime \prime}$ be the mass of the balancing poise, and $c^{\prime \prime}$ its distance from the geometrical center of the magnet, measured along the axis. Then in the above equations

$$
h=\frac{m^{\prime} h^{\prime}-m l}{m} \text { and } c=\frac{m^{\prime \prime} c^{\prime \prime}}{m}
$$

where $m$ equals the combined mass of magnet and poises.

If the magnet were suspended with knife-edge vertical and oscillated in a horizontal plane, the period would be

$$
T^{2}{ }_{k}=\frac{\pi^{2} m k^{2}}{M H}
$$

Using the equations (9) and (18) we have from (15)

$$
\epsilon=\frac{H T^{2} h}{T^{2}}\left(\frac{h^{2}+c^{2}+k^{2}}{k^{2}}\right) \theta_{r}
$$

or since $h$ and $c$ are small compared with $k$

$$
\epsilon=\frac{H T_{h}^{2} \theta_{r}}{T_{v}^{2}}
$$

Usually the scale-value is determined by deflecting at equal distances in the same relative positions both the $D$-variometer and the $Z$-variometer by an auxiliary magnet. Thus 


$$
\epsilon=\frac{H \tan u}{n}
$$

$u$ being the angular deflection of the $D$-variometer. Equation. (19) provides a method of determining $\epsilon$ by oscillations of the magnet in vertical and horizontal planes and is useful as a check on (20). As recording $Z$-magnets are surrounded by massive copper dampers, the oscillations in a vertical plane will require a correction for damping.

At the risk of digressing somewhat from the main subject, we may make the above equations for the periods and positions of equilibrium still more general and derive from them some interesting results. The equation of equilibrium (3) may be written

$$
\tan \theta_{0}=\frac{M F_{z}-G_{z} c}{M F_{x}-G_{z} h}
$$

in which $F_{x}, F_{y}, F_{z}$ are the magnetic forces, and $G_{x}, G_{y}$, and $G_{z}$ are the gravity forces along the coordinate axes. For any other set of rectangular axes equation (21) may be written

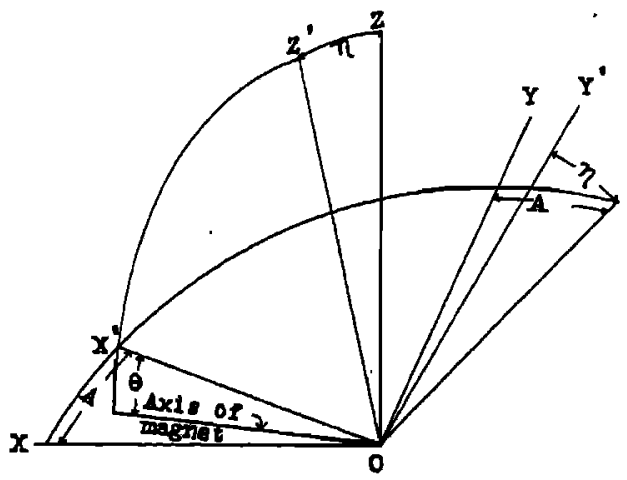

FIG. 2.

$$
\tan \theta_{0}=\frac{M F_{z^{\prime}}-G_{z^{\prime}} c}{M F_{x^{\prime}}-G_{z^{\prime}} h}
$$

in which $F_{x^{\prime}}, F_{y^{\prime}}$, and $F_{z^{\prime}}$ are the magnetic forces and $G_{x^{\prime}}$, $G_{y^{\prime}}$, and $G_{z^{\prime}}$ are the gravity forces along the new axes, $M$, $h$, and $c$ being the same as in the old axes. The two sets of axes are shown in Fig. 2.

The direction cosines between the axes are given in the following scheme:

\begin{tabular}{c|c|c|c}
\hline \hline & $X$ & $Y$ & $Z$ \\
\hline$X^{\prime}$ & $\cos \mathrm{A}$ & $\sin A$ & 0 \\
\hline$Y^{\prime}$ & $-\sin A \cos \eta$ & $\cos A \cos \eta$ & $\sin \eta$ \\
\hline$Z^{\prime}$ & $\sin A \sin \eta$ & $-\cos A \sin \eta$ & $\cos \eta$ \\
\hline
\end{tabular}


We have $F_{x^{\prime}}=F_{x} \cos \left(\mathrm{XX}^{\prime}\right)+F_{y} \cos \left(\mathrm{YX}^{\prime}\right)+F_{z} \cos \left(\mathrm{ZZ}^{\prime}\right)$, and similar formulae for the remaining new axes. For the gravity forces, $G_{x^{\prime}}=G_{x} \cos \left(\mathrm{XX}^{\prime}\right)+G_{y} \cos \left(\mathrm{YX}^{\prime}\right)+G_{z} \cos \left(\mathrm{ZX}^{\prime}\right)$ and similar formulae for the other two new axes. Since $F_{x}=H, F_{y}=0$, $-F_{\mathrm{z}}=-Z, G_{x}=0, G_{y}=0$, and $-G_{z}=-m g$, we have

$$
\left.\begin{array}{rlrl}
F_{x^{\prime}} & =H \cos A & G_{x^{\prime}} & =0 \\
F_{y^{\prime}} & =-H \sin A \cos \eta-Z \sin \eta & \mathrm{G}_{y^{\prime}} & =m g \sin \eta \\
-F_{z^{\prime}} & =-H \sin A \sin \eta+Z \cos \eta & -G_{z^{\prime}} & =-m g \cos \eta
\end{array}\right\}
$$

Substituting (23) in (22) noting that the forces $F_{y^{\prime}}$ and $G_{y^{\prime}}$ being perpendicular to the direction of motion, do not affect the motion of the magnet, we have

$$
\tan \theta_{0}=\frac{M(Z \cos \eta-H \sin A \sin \eta)-m g c \cos \eta}{M H \cos A-m g h \cos \eta}
$$

For $\eta=0$, (24) becomes equation (4) which has already been discussed. For $\eta=90^{\circ}, \tan \theta_{0}=\frac{-M H \sin A}{M H \cos A}=-\tan A$, and $\left(\theta_{0}+A\right)=0$. The magnet will be level and in the magnetic meridian, being sustained in a horizontal plane by the reactions at the knife-edge which is supposed to be replaced by suitable bearings. Suspending the magnet by a fibre at the point $O^{\prime}$ such that the center of gravity and the point $O^{\prime}$ are in the same vertical plane, $h$ will be 0 and we shall have the ordinary unifilar suspension. For $M=0$, we have the inclined compound pendulum and $\tan \theta_{0}=\frac{c}{h}$ (unstable position for positive value of $h$ and stable position for negative value of $h$ ); the center of gravity lies in the plane $Z O Z^{\prime}$ and the line $h$ makes the angle $\tan ^{-1} \frac{c}{h}$ with $O Z^{\prime}$. If $c=0, \theta_{0}=0$ and $h$ lies in the plane. $Z O Z^{\prime}$. For $M=0$ and $\eta=0, \tan \theta=\frac{c}{h}$; and for $c=0, \theta_{0}=0$ and $h$ is vertical downward and we have the ordinary compound pendulum. For $M=0$ and the angle $Z Z^{\prime}=90^{\circ}+\eta, \eta$ being a small angle, $\tan \theta_{0}=\frac{c}{h}$ and if $c=\theta, \theta_{0}=0, h$ lies in the plane $Z O 7^{\prime}$, and we have the horizontal pendulum. For $A=c=h=0$,

$$
\tan \theta_{0}=\frac{Z}{H} \cos \eta
$$


If $\eta=90^{\circ}$ the magnet would be horizontal and in the magnetic meridian. For $A=0$

$$
\tan \theta_{0}=\frac{(M Z-m g c) \cos \eta}{M H-m g h \cos \eta}
$$

According to equations (25) and (26) an error in $\theta_{0}$ due to an error in level, would depend on $\cos \eta, \eta$ being taken perpendicular to the magnetic meridian, and would be small or negligible if $\eta$ is small. According to equation (4) a displacement in $\theta_{0}$ due to an accidental displacement in $A$ by jarring the instrument would likewise be small for small displacements; and moreover according to equation (15) a change in scale-value due to the same cause would be small or negligible.

The general expression for the period of the magnet when it lies in the magnetic meridian, and is thus referred to the axes shown in Fig. 1, may be obtained from equation (7) by placing $A=0$. Thus

$$
T^{2}{ }_{a}=\frac{\pi^{2} K_{v}}{(M H-m g h) \cos \theta_{0}-(m g c-\overline{M Z}) \sin \theta_{0}}
$$

Referred to the new axes shown in Fig. 2 equation (27) may be written

$$
T^{2}{ }_{v}=\frac{\pi^{2} K_{v}}{\left(M F_{x^{\prime}}-G_{z^{\prime}} h\right) \cos \theta_{0}-\left(G_{z^{\prime}} c-M F_{z^{\prime}}\right) \sin \theta_{0}}
$$

Substituting from (23), the period referred to the new axes is

$T^{2}{ }_{v}=\frac{\pi^{2} K_{v}}{(M H \cos A-m g h \cos \eta) \cos \theta_{0}-[m g c \cos \eta-M(Z \cos \eta-H \sin A \sin \eta)]} \frac{\sin \theta_{0}}{(2)}$

For $\eta=0,(28)$ reduces to equation (7). For $\eta=90^{\circ}$ and hence $\theta_{0}=A=0$, as shown above, (28) reduces to (18); the magnet is level and moves in a horizontal plane; if it is suspended at the point $O^{\prime}$ by a fibre, $h=0$ and we have the ordinary unifilar suspension. For $M=c=0$, and so $\theta_{0}=0$, as shown above, we have an inclined compound pendulum oscillating around a fixed axis; its period is

$$
T^{2}=\frac{\pi^{2} K_{v}}{m g h \cos \eta}
$$

For $M=\eta=c=\theta_{0}=0$, we have the ordinary compound pendulum oscillating around a fixed horizontal axis. For $M=c=0$ and $Z Z^{\prime}=90^{\circ}+\eta, \eta$ being small, we have the period of the horizontal pendulum 
For $A=c=h=0$

$$
T^{2}{ }_{v}=\frac{\pi^{2} K_{v}}{m g h \eta}
$$

$$
T^{2}{ }_{v}=\frac{\pi^{2} K_{v}}{M H \cos \theta_{0}+M Z \cos \eta \sin \theta_{0}}
$$

which shows the effect of error in level. For $\eta=0$, this equation becomes (11) and for $\eta=90^{\circ}$, and so $\theta_{0}=0$, it becomes (18). For $A=0,(m g c-M Z)=0$ and so $\theta_{0}=0$

$$
T^{2}{ }_{v}=\frac{\pi^{2} K_{v}}{M H-m g h \cos \eta}
$$

which shows the error in period of the vertical-intensity variometer due to error in level. If $\eta=0$, this equation becomes (9).

We shall next take up the case where the magnet rolls on an axle of sensible diameter, $2 r$, for example the ordinary dip needle, and thus does not pivot or rotate around a fixed axis, as we have so far assumed. Since we are interested chiefly in the small oscillations, we may refer the center of gravity to the moving origin $P$ (Fig. 3). The coordinates of the center of gravity are $x=(a \sin \dot{\phi}-c \cos \phi)$ and $z=$ $(r+a \cos \phi+c \sin \phi)$ hence $\dot{x}^{2}+\dot{z}^{2}=$

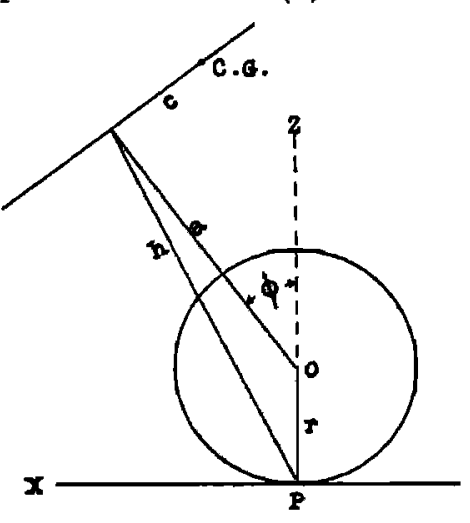

Fi6. 3. $\left(a^{2}+c^{2}\right) \dot{\phi}^{2}$. The equation of motion from (1) is

$$
\frac{d}{d t} \frac{d L}{d \phi}-\frac{d L}{d \phi}=0
$$

$L$, the kinetic potential, is equal to $T$, the kinetic energy, minus $V$, the potential energy. As before $T=\frac{m}{2}\left(\dot{x}^{2}+\dot{z}^{2}+k^{2} \dot{\phi}^{2}\right)=$ $\frac{m}{2}\left(a^{2}+c^{2}+k^{2}\right) \dot{\phi}^{2}$ and $V=m g(r+a \cos \phi+c \sin \phi)-M(H \cos \phi+$ $Z \sin \phi)$. Then from the equation of motion and after differentiation substituting $\sin \phi=\phi$ and $\cos \phi=1$, since $\phi$ is small, we have $m\left(a^{2}+c^{2}+k^{2}\right) \phi-(m g a-M H) \phi+m g c-M Z=0$. For the case when the magnet is level, since then $M Z=m g c$ and substituting the approximate value of $K_{v} \ddot{\phi}$ for the first term, we have 


$$
K_{v} \ddot{\phi}+(M H-m g a) \phi=0
$$

The relation between $a$ and $h$ is $h^{2}=a^{2}+r^{2}+2 a r \cos \phi$ and when $\phi$. is small

$$
a=h-r
$$

Eliminating $a$ by means of (31), (30) becomes

$$
K_{v} \phi+(M H-m g h+m g r) \phi=0
$$

and for rolling

$$
T^{2}{ }_{v}+\frac{\pi^{2} K_{v}}{M H-m g h+m g r}
$$

The work done in deflecting the pivoting magnet through a . small angle $\theta$ is $\int(M H-m g h) \theta d \theta=1 / 2(M H-m g h) \theta^{2}$; and the work done in deflecting the rolling magnet is $\int(M H-m g h+m g r) \phi d \phi$ $=\mathrm{x} / 2(M H-m g h+m g r) \phi^{2}$. When the magnet is deflected by another magnet as in scale-value deflections, the work done on it is the same whether it rolls or pivots. Hence

$$
(M H-m g h) \theta^{2}=(M H-m g h+m g r) \phi^{2}
$$

As the expression in the parenthesis on the left is smaller than the parenthesis on the right, $\theta$ is greater than $\phi$, or a pivoting magnet is more sensitive than a rolling magnet. The same conclusion would be reached by comparing the potential energies and the periods of the rolling and pivoting magnets. Writing (32) in the form

$$
\begin{aligned}
& \frac{M H-m g h+m g r}{M H-m g h}=\frac{\theta^{2}}{\phi^{2}} \text { or } \frac{m g r}{M H-m g h}=\frac{\theta^{2}-\phi^{2}}{\phi^{2}} \text { and } \\
& r=\left(\frac{M H-m g h}{m g}\right)\left(\frac{\theta^{2}-\phi^{2}}{\phi^{2}}\right)=\left(\frac{M \epsilon}{m g \theta_{r}}\right) \frac{\theta^{2}-\phi^{2}}{\phi^{2}}
\end{aligned}
$$

from which $r$ may be computed. For example, if magnet $J$ were adjusted to a scale-value of $5 \gamma$ per $\mathrm{mm}$. on the magnetogram at the distance $R=230 \mathrm{~cm}$. from the drum and showed the unequal deflections, $\phi=35 \mathrm{~mm}$. and $\theta=37 \mathrm{~mm}$. the angles being proportional to the deflections in mm., $r$ would be $0.0003 \mathrm{~cm} .=0.003 \mathrm{~mm}$. $=3 \mu$ if we assume that the magnet rolls in one direction and pivots in the other. If $r$ is not the same in both directions, there will be two different periods and likewise two different scale-values, according to the direction of the deflections.

There are causes of unequal deflections other than the unequal curvatures in the knife-edge, for example a magnet rolling on a 
plane inclined at a small angle. The discussion of this question would require the investigation of the potential energy function $V$ in the neighborhood of a minimum, and need not be considered here. For the motion of a body around a position of equilibrium is, in general, symmetrical up to the second powers of the displacements if they are small, and the motion of the magnet of a $Z$-variometer is of that type.

To obtain the equation of equilibrium of the rolling magnet, place $\frac{d V}{d \cdot \phi}=0, V$ being the complete form of the potential energy as given above. If the magnet is not in the magnetic meridian, we shall get

$$
\tan \phi=\frac{M Z-m g c}{M H \cos A-m g a}
$$

For the ordinary dip needle having an axle or pivots of sensible diameter the center of gravity coincides with the center of the pivots and $a=h-r=c=0$, whence

$$
\tan \phi=\frac{\tan I}{\cos A}
$$

which is the same as the equation (5) for the dip needle having pointed pivots.

It is a well-known phenomenon that magnets gradually lose their strength. Also the magnetic elements $D, H, Z$ undergo small changes from year to year called secular changes. Returning to equation (3)

$$
\theta_{0}=\frac{M}{M} \frac{Z-m g c}{H-m g h}
$$

$\theta_{0}$ would be expected to change gradually on account of changes in $M, Z$, and $H$. Regarding $Z$ as constant for the present, let us decide whether changes in $M$ or changes in $H$ are more important in affecting $\theta$. Dropping the relatively small term $m g h$ in the denominator, (3) may be. written $M H \theta=M Z-m g c$. Differentiating with reference to $M, H$, and $\theta$ and solving for $d \theta$

$$
d \theta=\left(\frac{Z}{\tilde{H}}-\theta\right) \frac{d M}{M}-\frac{\theta d H}{H}
$$

The last term on the right is very small, and hence the important agent in causing a change in $\theta$ is the gradual loss of magnetism of the magnet. This change in $\theta$ will appear in the gradual change or drift in the base-line values. Writing $M=M_{0}(1-\alpha t)$, where $\alpha$ is the coefficient of magnetic loss per annum, and noting that at $t_{0}=0, \theta_{0}=0, Z M-m g c=0$

$$
\triangle_{1} \theta=\frac{-Z M_{0} \alpha t}{H M_{0}(1-a t)-m g h}
$$

Using (15) and considering that $\triangle M=-M a t$, we have 


$$
\frac{\epsilon\left(\triangle_{1} \theta\right)}{Z \theta_{r}}=\frac{\triangle Z}{Z}=\frac{\triangle M}{M}
$$

or the apparent proportional change in $Z$ (the drift in base-line value) equals the proportional loss of magnetism in the magnet. For magnet $J$ mounted as a variometer and $\alpha=0.001$ and $\epsilon=5 \gamma$, the base-line drift would be $55 \gamma$ per annum, $Z$ being 0.554 .

It may be noted in passing that if $a$ be taken as the temperature coefficient (34) and (35) also hold for temperature changes.

To take the effect of changes in $Z$ into account, write $Z=Z_{0}(1-\beta t)$ and (34) will become, the products of small quantities being neglected,

$$
\triangle_{2} \theta=\frac{-Z M(a+\beta) t}{H M_{0}(1-a t)-m g h}
$$

The total drift, i. e. the total relative change in the position of the recording spot on the magnetogram, will be the combined effect of both loss of strength of the magnet and the secular change in $Z$, and the direction of the drift will be determined by the magnitude and sign of $\beta$.

Besides the vertical-intensity variometer having a single magnet as above, another type has a control magnet situated below the recording magnet with north end up. For this case

$$
\theta=\frac{M Z-\frac{2 M_{0} M_{0}^{\prime}}{r^{2}}-m g c}{M H-m g h}
$$

where $r$ is the distance and $M^{\prime}$ the magnetic moment of the control magnet. The control magnet makes no change in the formulae for period and scale-value of the recording magnet.

Letting $a$ and $\lambda$ be the coefficients of loss of magnetism in the recording and control magnets respectively, (37) will become

$$
\theta=\frac{Z M_{0}(1-a t)-\frac{2 M_{0} M_{0}^{\prime}}{r^{8}}[1-(\alpha+\lambda) t]-m g c}{H M_{0}(1-a t)-m g h}
$$

If we impose the condition $\left(Z M_{0}-\frac{2 M_{0} M_{0}^{\prime}}{r^{3}}-m g c_{1}\right)=0$ at time $t_{0}$ we have

$$
\Delta_{3} \theta=\frac{-M_{0} Z a t+\frac{2 M_{0} M_{0}^{\prime}}{r^{2}}(a+\lambda) t}{H M_{0}(1-a t)-m g h}
$$

Placing the numerator equal to zero and solving

$$
\frac{2 M_{0}^{\prime}}{r^{3}}=\frac{Z a}{a+1}
$$


which will determine the distance and the strength of the control magnet required to counteract the loss of magnetism in the recording magnet. If $\alpha=\lambda$, (40) will be

$$
\frac{M_{0}^{\prime}}{r^{3}}=\frac{Z}{4}
$$

If it were desired to take the secular change in $Z$ into account, then introducing $Z=Z_{0}(1-\beta t)$ into (39) and assuming the magnet was adjusted to be level at the start

$$
\Delta_{4} \theta=\frac{-Z_{0} M_{0}(a+\beta) \mathrm{t}+\frac{2 M_{0} M_{0}^{\prime}}{r^{3}}(a+\lambda) t}{H M_{0}(1-\alpha t)-m g h}
$$

and the condition for eliminating drift would be

$$
\frac{2 M_{0}^{\prime}}{r^{8}}=\frac{Z_{0}(a+\beta)}{(a+\lambda)}
$$

which of course reduces to (40) when $\beta=0$ and to (41) when $a=\lambda$ also.

Equation (43) indicates the possibility of designing a verticalintensity variometer which would be practically free from drift for any place where $\beta$ is known, provided magnets possessing the required coefficients $\alpha$ and $\lambda$ could be made or selected. The condition that $\alpha=\lambda$ could possibly be realized in practice by making the magnets from the same piece of steel, and magnetizing them in the same magnetizing coil at the same field intensity.

Let us assume $\alpha=\lambda$ and compute the strength of control magnet required to counteract the drift due to loss of magnetism, magnet $J$ being used as a $Z$-variometer. Applying (41), the coefficients of loss of magnetism being equal, the control magnet $M^{\prime}$ at a distance of $14 \mathrm{~cm}$. below, would have to be about 0.5 the size or volume of magnet $J$; moreover, if the temperature-coefficients of the magnets were equal the variometer would be compensated for temperature changes, and the recorded variations would require no correction for temperature. Thus a vertical-intensity variometer may, by means of a control magnet, be compensated either for drift in base-line values, or for temperature changes, provided the coefficients of loss of magnetism and the temperature coeffcients are the same for both the recording magnet and the control magnet. This compensation can be effected by the appropriate adjustment of the distances $c$ and $r$.

If $a=\lambda$, the control magnet can be separated into two portions

$$
M_{0}^{\prime}=B+D
$$

where $B=\frac{Z r^{3}}{4}$ from (41) and is that portion necessary to neutralize the drift due to loss of magnetism. Then using (44), (43) becomes 


$$
\frac{D}{B}=\frac{\beta}{\alpha} \text { or } D=\frac{Z r^{3} \beta}{4 \alpha}
$$

$D$ is that portion of the control magnet effective in counteracting the secular change in $Z$. For example, for a secular decrease in $Z$ of some $55 \gamma$ per annum and so $\beta=0.001$, a control magnet of about one half the size or volume of magnet $J$ would be sufficient to counteract the decrease in $Z$ : and in the example above, the same size of control magnet is sufficient to counteract the loss of magnetism. Hence, a control magnet of the same size as magnet $J$ would be sufficient, under the conditions assumed, to counteract both loss of magnetism and of secular decrease in $Z$. If this control magnet were closer, or larger, the variometer would be over-compensated and the recording spot of light would drift upward on the magnetogram or in the positive direction of $\theta$. On the other hand, if the control magnet were farther away, or smaller, undercompensation would ensue and the spot would drift downward in the negative direction of $\theta$.

Besides movements of the magnet arising from magnetic changes, $Z$-variometers are sometimes subject to abrupt mechanical displacements. They are most likely to occur when the instrument is jarred, or when, as in making scale-value deflections, the deflecting magnet is brought up to or taken away from the instrument too quickly. In displacements due to jars, taking into consideration that the weight of the magnet is concentrated in the very small area of contact between the knife-edge and the agate plane, and that the pressure is thus very great, the knife-edge is probably injured or fractured and obstructed by the dust particles thrown in its way. Displacements or shifts due to deflecting the magnet indicate an imperfect knife-edge.

We may say, then, that the most important operating characteristics of the $Z$-variometer are: stability, sensitivity, and freedom from shifts. It would be of theoretical interest to investigate how these three characteristics are affected by imperfections in the knife-edge (fractured surfaces, for example) and by dust particles or other obstructions. However, it is clear these characteristics are vastly impaired by imperfections in the knife-edge; and on the other hand, both theory and experience have shown they may be secured by means of an accurately ground knife-edge.

In conclusion, it will probably be needless to emphasize the fact that a vertical-intensity variometer is an instrument of the greatest delicacy, and requires the utmost care in its operation. And in order that it may faithfully perform its function to record intensity changes of extreme minuteness, the knife-edge-the most important mechanical part of the instrument-must be maintained in the most perfect condition possible.

\footnotetext{
U. S. Coast and Geodetic Survey, Cheltenham Magnetic Observatory, May, 1918.
} 